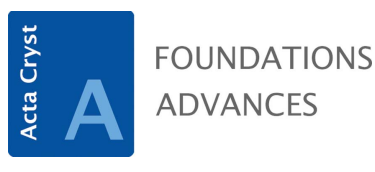

ISSN 2053-2733

Keywords: thermoelectricity; transport properties; magnetic order; galvanomagnetic effects; thermomagnetic effects

Supporting information: this article has supporting information at journals.iucr.org/a

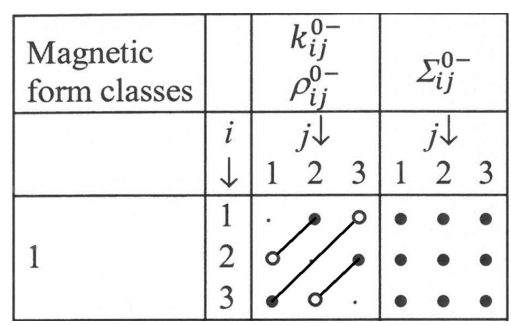

C 2020 International Union of Crystallography

\section{Thermoelectric transport properties in magnetically ordered crystals. Further corrigenda and addenda}

\author{
Hans Grimmer* \\ Multiscale Materials Experiments, Research with Neutrons and Muons, Paul Scherrer Institut, Forschungsstrasse 111, \\ Villigen PSI, CH-5232, Switzerland. *Correspondence e-mail: hans.grimmer@psi.ch
}

Further corrigenda and addenda for the article by Grimmer [Acta Cryst. (2017), A73, 333-345] are reported. New figures in the supporting information show how the restrictions on the forms of galvanomagnetic and thermomagnetic tensors are related to those on corresponding thermoelectromagnetic tensors.

Corrigenda. Corrections to Grimmer (2017) are needed in Figs. 4(c) and 6(c) for the fourth-rank tensors containing components marked in blue and in Fig. $6(b)$ for $\rho_{i j l m}^{-}, k_{i j l m}^{-}$in magnetic form class $4^{\prime}$. The corrections are included in the figures in the supporting information.

Addenda. For even tensors (invariant under space inversion $\overline{1}$ and time inversion $1^{\prime}$ ), the galvanomagnetic tensors $\rho$ and the thermomagnetic tensors $k$ are symmetric in the first two indices if the rank is even and antisymmetric if the rank is odd, whereas the thermoelectromagnetic tensors $\Sigma$ are general for all ranks. For magnetic tensors (invariant under $\overline{1}$ and changing sign under $1^{\prime}$ ), $\rho$ and $k$ are antisymmetric in the first two indices if the rank is even and symmetric if the rank is odd, whereas the tensors $\Sigma$ are general for all ranks. The intrinsic symmetry of these tensors has been discussed in more detail by Gallego et al. (2019).

Exchanging $\bullet$ and $\circ$ for certain components, new figures are obtained that clearly show how the forms of the tensors for $\rho$ and $k$ are related to those for $\Sigma$. The forms of the tensors for the limit point groups described in Grimmer (2019) are included in the supporting information in Figs. 1-6.

\section{References}

Gallego, S. V., Etxebarria, J., Elcoro, L., Tasci, E. S. \& Perez-Mato, J. M. (2019). Acta Cryst. A75, 438-447.

Grimmer, H. (2017). Acta Cryst. A73, 333-345.

Grimmer, H. (2019). Acta Cryst. A75, 409. 